\title{
AUTOMORPHISM GROUPS OF FREE GROUPS
}

\author{
M. F. NEWMAN \\ (Received 21 February 2008; accepted 4 May 2008) \\ Communicated by Peter M. Neumann \\ Dedicated to Cheryl Praeger for her sixtieth birthday
}

\begin{abstract}
This note contains some remarks on generating pairs for automorphism groups of free groups. There has been significant use of electronic assistance. Little of this is used to verify the results.
\end{abstract}

2000 Mathematics subject classification: primary 20F28; secondary 20E05.

Keywords and phrases: automorphism groups, free groups, computation.

\section{Introduction}

As Chandler and Magnus [CM82] point out in their history of combinatorial group theory, the study of automorphisms of free groups played a significant role in the early development of the theory, especially the work of Nielsen in a number of papers from 1917 to 1924 (see [Nie86]). The culmination of this work of Nielsen was finite presentations for these automorphism groups. His presentations have four generators; and three generators when the free group has rank two. Neumann started his research career in the early 1930s by finding presentations with two generators when the free groups have rank at least four; and three generators when the free group has rank three [Neu32].

The study of these automorphism groups has continued. For example, there is a report on cohomology in a lecture that Vogtmann gave at the ICM2006 [Vog06] and an account of work on linear representations by Grunewald and Lubotzky [GL06]. Work has also continued on generating sets and presentations. In particular, Tamburini and Wilson [TW97] have shown that the automorphism groups of free groups with rank at least 18 can be $(2,3)$-generated; there are generating pairs of automorphisms with orders 2 and 3. In this connection Vsemirnov [Vse07] has recently shown that $\mathrm{GL}(6, \mathbb{Z})$ can be $(2,3)$-generated. There is a new approach to presentations

(c) 2009 Australian Mathematical Society 1446-7887/09 \$A2.00+0.00 
in [AFV08]. The presentations of Nielsen (1924) are built from presentations of the relevant symmetric group by adding two generators and 14 relations. So the recent work on presentations of symmetric groups (see [BCLGO08] and [GKKL08]) gives further presentations for free groups.

The first aim of this note is to fill the gap evident above by showing that the automorphism groups of the free groups of rank two and three can in fact be generated by two elements (Theorems 1 and 3).

The generating pair found for the rank-three case consists of automorphisms with order three and order four. Both automorphisms in the generating pair found for the rank-two case have infinite order. In fact, all generating pairs for the rank-two case consist of automorphisms with infinite order (Theorem 2).

The generating pairs are also minimal in another sense. The length of an automorphism of a free group is the sum of the lengths of the images of a free generating set. The automorphisms of minimal length are the signed permutations that form a finite subgroup of the automorphism group. For the automorphism group of a free group with rank $n$, the generating pairs of Tamburini and Wilson consist of a permutation with order three and an automorphism with order two and length $n+1$. The generating pairs of Neumann for rank $n$ consist of an automorphism with order 12 and length $n+1$ and either a permutation with order $n$ for even rank at least four, or a signed permutation with order $2 n$ for odd rank at least five. The generating pair in Theorem 3 consists of a signed permutation with order four and an automorphism with order three and length four. The automorphisms in the generating pair in Theorem 1 have length three. This is minimal as a corollary of Theorem 2.

Finding these results made use of the computer algebra system MAGMA [BCP97], but most of the proofs given do not rely on the use of computers.

\section{Two-generator case}

THEOREM 1. The group of automorphisms of a free group of rank two can be generated by two automorphisms.

PROOF. Let $\{a, b\}$ be a free generating set for the underlying free group $F_{2}$. Denote the automorphism that maps $a$ to $u$ and $b$ to $v$ by $[u, v]$. Nielsen proved that $\operatorname{Aut}\left(F_{2}\right)$ is generated by $P=[b, a], O=\left[a^{-1}, b\right]$ and $U=[a b, b]$. It suffices to show the automorphisms $U$ and $V=\left[a^{-1} b, a\right]$ generate $\operatorname{Aut}\left(F_{2}\right)$. It is routine to check that $O=U^{-1} V^{-1} U V V$ and $P=O U^{-1} O V$.

MAGMA has proved very helpful for finding a suitable pair of automorphisms. Briefly, a generating pair was eventually found as follows. Neumann listed six relations that hold among the three generators, $P, O, U$. A systematic search through pairs of words in $P, O, U$ of length up to four shows that the subgroup generated by $\{U, V=O U O P\}$ has index one (an easy coset enumeration). A search through the automorphisms generated by all group words in $U, V$ with word length at most five finds $O$. 
Notice that the two automorphisms $U, V$ have infinite order. In the light of the result of Tamburini and Wilson, it is natural to ask whether there are generating pairs in which at least one automorphism has finite order.

THEOREM 2. In every generating pair for $\operatorname{Aut}\left(F_{2}\right)$ both automorphisms have infinite order.

It took quite some searching with MAGMA to find the group $H$ in the proof.

PROOF. The conjugacy classes of elements in $\operatorname{Aut}\left(F_{2}\right)$ with finite order have been determined by Meskin [Mes74]. In terms of $P, O, U$, his set of representatives of these classes is $\{\mathrm{Id}, P, O, P O P O, O P O U O U P, U P O, P O\}$. Let $H$ be the group defined by

$$
\begin{array}{r}
\langle\bar{P}, \bar{O}, \bar{U}| \bar{P}^{2}, \bar{O}^{2},(\bar{P} \bar{O})^{4},[\bar{U}, \bar{O} \bar{U} \bar{O}],(\bar{P} \bar{O} \bar{P} \bar{U})^{2},(\bar{U} \bar{P} \bar{O})^{3} \\
\left.(\bar{U} \bar{P} \bar{U} \bar{O})^{2},\left(\bar{P} \bar{U}^{4}\right)^{2},(\bar{P} \bar{U})^{6}\right\rangle
\end{array}
$$

This is a finite group with order 576. It follows from a presentation for $\operatorname{Aut}\left(F_{2}\right)$ on $P, O, U$ (see [MKS66, Problem 2 of Section 3.5]) that the group $H$ is a quotient of Aut $\left(F_{2}\right)$ under $\theta: P \mapsto \bar{P}, O \mapsto \bar{O}, U \mapsto \bar{U}$. So it suffices to check for each class representative $v$ above and each element $h$ of $H$ that the subgroup of $H$ generated by $\{v \theta, h\}$ is proper. Use of MAGMA verifies this easily.

Note that $\operatorname{GL}(2, \mathbb{Z})$ has a generating pair with an element of order two (see Coxeter and Moser [CM80, Section 7.2]). Since the automorphisms with length two are the signed permutations and have finite order, both automorphisms in a generating pair for $\operatorname{Aut}\left(F_{2}\right)$ have length at least three. So the generating pair in Theorem 1 has minimal length.

\section{Three-generator case}

THEOREM 3. Aut $\left(F_{3}\right)$ can be generated by a pair of automorphisms.

Again, finding a generating pair made use of MAGMA. The method used for the two-generator case, pushed harder and refined, worked.

PROOF. Let $\{a, b, c\}$ be a free generating set for $F_{3}$. Denote the automorphism that maps $a$ to $u, b$ to $v$ and $c$ to $w$ by $[u, v, w]$. Nielsen proved that $\operatorname{Aut}\left(F_{3}\right)$ is generated by $P=[b, a, c], Q=[b, c, a], O=\left[a^{-1}, b, c\right]$ and $U=[a b, b, c]$.

Let $r, s$ be the automorphisms defined by

$$
r=\left[b, a^{-1}, c^{-1}\right], \quad s=\left[a, c^{-1}, b c^{-1}\right] .
$$

It is straightforward to check that the Nielsen generators can be expressed in terms of $r, s$ as follows: 


$$
\begin{aligned}
& P=r s r s r s^{2} r s^{2} s^{2} r s r s^{2} r s^{2}, \\
& Q=s r s^{2} r s r^{2} s^{2} r^{2} s r^{3} s r^{3} s^{2} r^{3} s r^{3} s^{2} r s^{2} r^{3} s r s^{2} r, \\
& O=r s r s^{2} r s^{2} r^{3} s r s r s r^{3} s r^{3} s^{2} r^{3} s^{2} r s r^{3} s^{2}, \\
& U=r^{2} s r s r s^{2} r s r^{2} s^{2} r s r s r s r^{3} s r^{3} s^{2} r^{3} s r^{3} .
\end{aligned}
$$

Note that the two automorphisms $r, s$ have order four and three respectively and length three and four respectively. As before, this sum of lengths is minimal. There are also generating pairs consisting of a permutation with order three and an automorphism with order four and length four. Note that $\operatorname{Aut}\left(F_{3}\right)$ is not $(2,3)$ generated (see [TZ00], [Nuz01] or The Kourovka Notebook [MK06, 14.49]), or use that $\operatorname{Aut}\left(F_{3}\right)$ maps onto the affine general linear group $\operatorname{AGL}(3,2)$, which is not $(2,3)$ generated.

Finally, is $\operatorname{Aut}\left(F_{3}\right)(2,4)$-generated or not?

\section{Acknowledgements}

I am indebted to Professors Conder, Grunewald, Tamburini and Vogtmann for answering questions in the search for relevant results in the literature, and Laci Kovács for helpful conversations.

\section{References}

[AFV08] H. Armstrong, B. Forrest and K. Vogtmann, 'A presentation for $\operatorname{Aut}\left(F_{n}\right)$ ', J. Group Theory 11(2) (2008), 267-276.

[BCP97] W. Bosma, J. Cannon and C. Playoust, 'The Magma algebra system. I. The user language', J. Symbolic Comput. 24(3-4) (1997), 235-265 (Computational Algebra and Number Theory, London, 1993).

[BCLGO08] J. N. Bray, M. D. E. Conder, C. R. Leedham-Green and E. A. O'Brien, 'Short presentations for alternating and symmetric groups', Preprint, 2008.

[CM82] B. Chandler and W. Magnus, The History of Combinatorial Group Theory, Studies in the History of Mathematics and Physical Sciences, 9 (Springer, New York, 1982).

[CM80] H. S. M. Coxeter and W. O. J. Moser, Generators and Relations for Discrete Groups, 4th edn, Ergebnisse der Mathematik und ihrer Grenzgebiete, 14 (Springer, Berlin, 1980).

[GL06] F. Grunewald and A. Lubotzky, 'Linear representations of the automorphism group of a free group', Preprint, 2006, ArXiv:math0606182. Geom. Funct. Anal. accepted.

[GKKL08] M. R. Guralnick, W. M. Kantor, M. Kassabov and A. Lubotzky, 'Presentations of finite simple groups: a quantitative approach', J. Amer. Math. Soc 21(3) (2008), 711-744.

[MKS66] W. Magnus, A. Karrass and D. Solitar, Combinatorial Group Theory: Presentations of Groups in Terms of Generators and Relations (Wiley Interscience, New York, 1966).

[MK06] V. D. Mazurov and E. I. Khukhro (eds.). The Kourovka Notebook, 16th edn (Russian Academy of Sciences, Siberian Division, Institute of Mathematics, Novosibirsk, 2006) (Unsolved problems in group theory, including archive of solved problems).

[Mes74] S. Meskin, 'Periodic automorphisms of the two-generator free group', in: Proc. 2nd Int. Conf. on the Theory of Groups, Australian National University, Canberra, 1973, Lecture Notes in Mathematics, 372 (Springer, Berlin, 1974), pp. 494-498.

[Neu32] B. Neumann, 'Die Automorphismengruppe der freien Gruppen', Math. Ann. 107 (1933), 367-386. (Published 1932.) 
[Nie86] J. Nielsen, Jakob Nielsen: Collected Mathematical Papers, Contemporary Mathematicians, 2 (Birkhäuser, Boston, MA, 1986), Edited and with a preface by Vagn Lundsgaard Hansen.

[Nuz01] Ya. N. Nuzhin, 'On a question of M. Conder', Mat. Zametki 70(1) (2001), 79-87.

[TW97] M. C. Tamburini and J. S. Wilson, 'On the $(2,3)$-generation of automorphism groups of free groups', Bull. London Math. Soc. 29(1) (1997), 43-48.

[TZ00] M. C. Tamburini and P. Zucca, 'On a question of M. Conder', Atti Accad. Naz. Lincei Cl. Sci. Fis. Mat. Nat. Rend. Lincei (9) Mat. Appl. 11(1) (2000), 5-7.

[Vog06] K. Vogtmann, 'The cohomology of automorphism groups of free groups', in: International Congress of Mathematicians, II, Madrid, 2006 (European Mathematical Society, Zürich, 2006), pp. 1101-1117.

[Vse07] M. Vsemirnov, 'The group GL(6, Z) is (2,3)-generated', J. Group Theory 10(4) (2007), 425-430.

M. F. NEWMAN, Mathematical Sciences Institute, Australian National University, ACT 0200, Australia

e-mail: newman@maths.anu.edu.au 